\title{
Atelocollagen-mediated siRNA delivery: future promise for therapeutic application
}

Keywords: atelocollagen $\bullet$ cancer $\bullet$ drug delivery $\bullet$ nucleic acid-based medicine $\bullet$ siRNA

\section{Drug-delivery systems for nucleic} acid-based medicine

In recent years, nucleic acid-based medicine has attracted attention for its therapeutic applications to cancers and infectious, hereditary, and other diseases. Moreover, treatment by gene transfer with a viral or nonviral vector has been actively studied. In addition, owing to the development of novel gene-transfer reagents and gene systems, the administration of a gene itself has been tested for potential therapeutic efficacy in gene expression. Most recently, the discovery of RNAi has triggered a global competition among many companies and researchers to find effective delivery systems that capitalize on the clinical benefits of siRNA.

For in vivo delivery of nucleic acids including siRNA, research and development have focused on the improvement of delivery efficiency, resulting in a wide use of genedelivery systems based on cationic liposomes or polymers. However, these delivery systems still entail clinically relevant problems such as cellular toxicity and the induction of nonspecific immune responses, as the expression of immune-responsive genes and apoptosisrelated genes is enhanced after administration. Establishment of an ideal delivery system with lower toxicity and higher stability is a big challenge [1-10].

To solve these problems, we and others have pioneered in vivo nucleic acid-delivery systems, such as siRNA systemic delivery mediated by highly bio-compatible and lowimmunogenic atelocollagen, which many researchers have extensively employed in studies of various disease models. We also investigated the combined use of siRNA/ atelocollagen with anticancer agents, targeting the antiapoptotic factor $B c l-x L$ gene. In brief, an siRNA targeting human $B c l-x L$ complexed with atelocollagen was injected three-times weekly for a total of 12-times through the caudal vein in mice with a human prostate cancer cell line (PC-3) xenograft already grown subcutaneously. These injections significantly inhibited growth as well as the expression of both Bcl-xL mRNA and its protein in tumors. A combinational therapy of $\mathrm{Bcl}-\mathrm{xL}$ siRNA/atelocollagen with a low dose of cisplatin $(12 \mathrm{mg} / \mathrm{kg})$ provided more intense growth inhibition [11].

Atelocollagen has been developed for artificial organs, its safety having been assured as a top-level bio-compatible material by the US FDA. Atelocollagen possesses basicity (positive charge) under physiological conditions, whereas siRNA and other nucleic acids have acidity (negative charge). Therefore, atelocollagen and siRNA are considered to form a complex by electrostatic binding. The nucleic acid complexed with atelocollagen can avoid degradation by nuclease(s) and can be efficiently transported in the bloodstream to tissues and cells, where the siRNA/ atelocollagen complex may be incorporated through endocytosis. Furthermore, recent studies demonstrated that the complex itself can lower the cellular membrane potential enough to promote endocytosis. In animal model experiments, the siRNA/atelocollagen complex that arrived at a tumor tissue downregulated the target gene expression. Accordingly, expectations are growing for the siRNA/atelocollagen complex as a novel

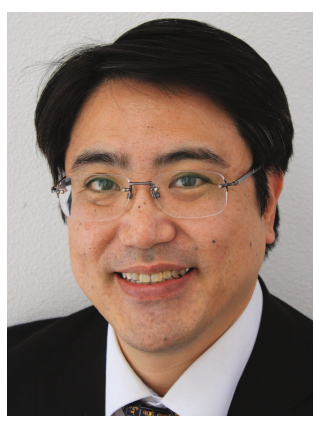

Ichiro Fujimoto Koken Research Institute, Koken Co. Ltd, Tokyo, Japan

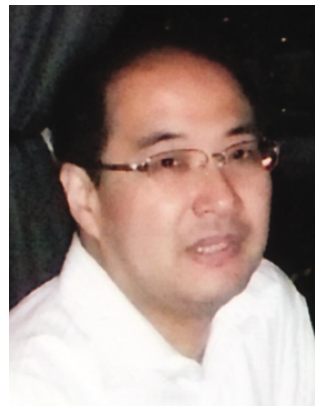

Yoshifumi Takei

"Author for correspondence: Division of Disease Models, Center for Neurological Diseases \& Cancer, Nagoya University Graduate School of Medicine, 65 Tsurumai-cho, Showa-ku, Nagoya 466-8550, Japan

and

Department of Biochemistry, Nagoya University Graduate School of Medicine, Nagoya, Japan

Tel.: +81527442060

Fax: +81 527442065

takei@med.nagoya-u.ac.jp

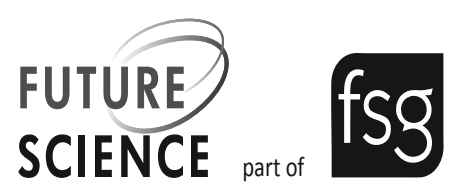


sustained release preparation for nucleic acid-based medicine [12].

\section{Preventing off-target effects for innate immunoreactivity of siRNA}

A great deal of attention was generated by a report that showed activation of the innate immune system by double-stranded RNA molecules, rather than by sequencespecific translation suppression as we expected, had a therapeutic effect on age-related macular degeneration, which is a top focus of siRNA drug discovery [13]. Soon, it was proved that once siRNA binds to the nucleic-acid recognizing sensor receptor TLR3 on the surface of vascular endothelial cells, IL-12 and IFN- $\gamma$ are secreted, causing the intense inhibition of vascularization. Then Opko Health, Inc., (FL, USA) a multinational pharmaceutical and diagnostics company that had started on a Phase III clinical trial of siRNA, faced a sharp decline in its own stock price and discontinued the trial. More recently, various approaches have been found to prevent the activation of innate immunity by devising a nucleic acid sequence and/or a chemical modification process, although experiments toward this end must be designed carefully.

Recently, experiments in which age-related macular degeneration model mice were similarly prepared via laser irradiation showed that the atelocollagen drug delivery system (DDS) does not cause the off-target effect [14]. Among the atelocollagen DDSs, the control siRNA did not suppress vascularization; VEGF siRNA offered sequence-specific therapeutic effects that are confirmable along with the siRNA incorporated into the retinal pigment epithelium.

Furthermore, we used siRNAs carrying type I IFN inducible sequences to evaluate IFN and inflammatory cytokine responses to various nucleic acid DDSs, including an atelocollagen DDS. The atelocollagen DDS exhibited no immune activation when administered to $\mathrm{Balb} / \mathrm{c}$ mice or added to human peripheral blood monocyte cultures, whereas a cationic lipid DDS enhanced inflammatory cytokine responses (IFN- $\alpha$, TNF- $\alpha$, IL-1 $\beta$, IL- 6 and IL-12) [15]. We also demonstrated the therapeutic effectiveness of systemic atelocollagen-mediated delivery of a siRNA targeting MCP-1, a leukocyte chemotactic factor, in the treatment of contact hypersensitivity of the skin [7]. In contact hypersensitivity model mice, intravenous administration of MCP-1 siRNA/atelocollagen significantly reduced the expression of MCP-1 protein at the inflammationinduced site and effectively inhibited the infiltration of monocytes, macrophages, and $\mathrm{T}$ cells, leading to a favorable outcome for inflammation-induced auricular swelling [7]. These results indicate that atelocollagen is an excellent vehicle for nucleic acid DDSs.

\section{Atelocollagen is safe in living bodies}

To assess the biotoxicity and related risk of a drug for systemic administration, various studies including acute toxicity testing, antigenicity testing, and carcinogenicity testing have been conducted. However, every test requires extraordinary time and labor. In recent years, DNA microarray procedures have been preferred as simpler and more efficient alternatives to other procedures for analyzing the toxicity and efficacy of a drug [16]. A DNA microarray can analyze tens of thousands to hundreds of thousands of DNA spots simultaneously to measure the transcription amount of each gene. Therefore, the microarray technique enables efficient and comprehensive analyses of gene behaviors. These characteristics of this technology promise broad utilization in clinical settings and testing of drugs and food additives [17].

Indeed, using DNA microarray technology, we compared gene expression in the liver of mice $24 \mathrm{~h}$ after administration of atelocollagen or a cationic polymer (both commercially available agents to introduce nucleic acids in vivo) through the caudal vein. The toxicity of the cationic polymer versus a lower toxicity of atelocollagen was quantitatively verified. In the cationic polymer group, apoptosis- or inflammatoryresponsive, cell-toxic genes have increased in number to more than 100-times or at least to 2.04-times; in contrast, such genes no more than doubled in the atelocollagen group. These findings indicate that atelocollagen is biologically safe even at a very high level [18].

\section{Application of atelocollagen as a vehicle for sustained release of drugs}

For DDS preparations, it is important to deliver a drug to the target tissue(s) at an effective dose over many hours.

Studies are now focusing on administration methods and dosage forms that minimize adverse drug reactions and enhance drug efficacy as much as possible. Successful DDS preparations will be obtained after these questions are answered: what materials should be used and how should a drug be delivered and slowly released?

Atelocollagen can be shaped into any form and, after the drug is released, atelocollagen is absorbed, metabolized and finally degraded in vivo. These properties are favorable for a base material of DDS. Therefore, atelocollagen can be used not only in solution, but also in sheet, sponge, powder, and other forms, providing a broad range of clinical applications.

Administration through the caudal vein has delivered many nucleic acids/drugs to tumors, owing to an enhanced permeability and retention effect, although poor delivery to many other nontarget 
normal tissues and organs has also been simultaneously observed. One of the delivery related problems in the treatment of a disease is the need to select a method of delivering only to the target organ while lessening effects on normal organs and tissues as much as possible. In this regard, there is still a high hurdle to be jumped for the systemic administration of not only atelocollagen but also many other DDS vehicles in clinical application. The development of atelocollagen with organ-specific modifications and other devices will lead to a bright future of clinical application to humans.

\section{References}

1 Takei Y, Kadomatsu K, Yuzawa Y, Matsuo S, Muramatsu T. A small interfering RNA targeting vascular endothelial growth factor as cancer therapeutics. Cancer Res. 64(10), 3365-3370 (2004).

2 Hanai K, Kurokawa T, Minakuchi Y. Potential of atelocollagen-mediated systemic antisense therapeutics for inflammatory disease. Hum. Gene Ther. 15(3), 263272 (2004).

3 Nakamura M, Ando Y, Nagahara S. Targeted conversion of the transthyretin gene in vitro and in vivo. Gene Ther. 11(10), 838-846 (2004).

4 Takeshita F, Minakuchi Y, Nagahara S et al. Efficient delivery of small interfering RNA to bone-metastatic tumors by using atelocollagen in vivo. Proc. Natl Acad. Sci. USA 102(34), 12177-12182 (2005).

5 Nakazawa K, Nemoto T, Hata T et al. Single-injection ornithine decarboxylase-directed antisense therapy using atelocollagen to suppress human cancer growth. Cancer 109(5), 993-1002 (2007).

6 Matoba T, Orba Y, Suzuki T et al. An siRNA against JC virus (JCV) agnoprotein inhibits JCV infection in JCV-producing cells inoculated in nude mice. Neuropathology 28(3), 286-294 (2008).

7 Ishimoto T, Takei Y, Yuzawa Y et al. Downregulation of monocyte chemoattractant protein-1 involving short interfering RNA attenuates hapten-induced contact hypersensitivity. Mol. Ther. 16(2), 387-395 (2008).

8 Ashihara E, Kawata E, Nakagawa Y et al. Beta-catenin small interfering RNA successfully suppressed progression of multiple myeloma in a mouse model. Clin. Cancer Res. 15(8) 2731-2738 (2009).

9 Sasaki T, Nakashiro K, Tanaka H et al. Knockdown of Akt isoforms by RNA silencing suppresses the growth of human

\section{Financial \& competing interests disclosure}

This work was supported by Grants-in-Aid from the Ministry of Education, Culture, Sports, Science, and Technology of Japan (17016030) and from the Japan Society for the Promotion of Science (17790185, 19590273, 21590305, and 24590348). The authors have no other relevant affiliations or financial involvement with any organization or entity with a financial interest in or financial conflict with the subject matter or materials discussed in the manuscript apart from those disclosed

No writing assistance was utilized in the production of this manuscript.

prostate cancer cells in vitro and in vivo. Biochem. Biophys. Res. Commun. 399(1), 79-83 (2010).

10 Takeshita F, Takahashi RU, Onodera J, Ochiya T. In vivo imaging of oligonucleotide delivery. Methods Mol. Biol. 872, 243-253 (2012).

11 Mu P, Nagahara S, Makita N, Tarumi Y, Kadomatsu K, Takei Y. Systemic delivery of siRNA specific to tumor mediated by atelocollagen: combined therapy using siRNA targeting $\mathrm{Bcl}-\mathrm{xL}$ and cisplatin against prostate cancer. Int J. Cancer. 125(12), 2978-2990 (2009).

12 Hanai K, Kojima T, Ota M, Onodera J, Sawada N. Effects of atelocollagen formulation containing oligonucleotide on endothelial permeability. J. Drug Deliv. 245835 (2012), (2012).

13 Kleinman ME, Yamada K, Takeda A et al. Sequence- and target-independent angiogenesis suppression by siRNA via TLR3. Nature 452, 591-597 (2008).

14 Ito Y, Nozaki M, Sugitani K, Ashikari M, Hirano Y, Ogura Y. Efficient delivery of siRNA by atelocollagen in a murine laser-induced choroidal neovascularization model. Ophthalmologica 230(4), 215-221 (2013).

15 Inaba S, Nagahara S, Makita N et al. Atelocollagen-mediated systemic delivery prevents immunostimulatory adverse effects of siRNA in mammals. Mol. Ther. 20(2), 356-366 (2012).

16 Jain KK. Applications of biochip and microarray systems in pharmacogenomics. Pharmacogenomics 1(3), 289-307 (2000).

17 Hardiman G. Applications of microarrays and biochips in pharmacogenomics. Methods Mol. Biol. 448, 21-30 (2008).

18 Ogawa S, Onodera J, Honda R, Fujimoto I. Influence of systemic administration of atelocollagen on mouse livers: an ideal biomaterial for systemic drug delivery. J. Toxicol. Sci. 36(6), 751-762 (2011). 